\title{
The Effects of a Bridging Exercise Applying Changes in the Base of Support for the Shoulders on Trunk Muscle Activation
}

\author{
Tae-Gyu Lee, PT $\cdot$ Chan-Hyun Park $\cdot H o-H e e$ Son, PT, PhD ${ }^{1 \dagger}$ \\ Department of Physical Therapy, Graduate School, Catholic University of Pusan \\ ${ }^{1}$ Department of Physical Therapy, College of Health Science, Catholic University of Pusan
}

Received: July 7, 2016 / Revised: July 18, 2016 / Accepted: August 10, 2016

(C) 2016 J Korean Soc Phys Med

\begin{abstract}
| Abstract |
PURPOSE: Bridge exercise is widely used in rehabilitation exercise for trunk stabilization through various applications in clinical practice. However, there is a lack of studies changing the base of support for the shoulders. The purpose of this study is to investigate the changes in the base of support for the shoulders of trunk muscle activation during bridge exercise.
\end{abstract}

METHODS: 20 healthy subjects ( 10 men, 10 women) in their twenties were participated in this study. They performed 5 bridge exercises (bridge exercise with their shoulders on a stable table ( $1 / 2$ knee height, knee height), and on a sling ( $1 / 2$ knee height, knee height), conventional bridge exercise. The surface electromyography were used for rectus abdominis (RA), internal oblique (IO), external oblique (EO), and erector spinae (ES).

RESULTS: During bridge exercise that their shoulders on the sling of $1 / 2$ knee height, the RA, EO, IO muscle activities were significant increased. And during bridge exercise that

†Corresponding Author : sonhh@cup.ac.kr

This is an Open Access article distributed under the terms of the Creative Commons Attribution Non-Commercial License (http://creativecommons.org/licenses/by-nc/3.0) which permits unrestricted non-commercial use, distribution, and reproduction in any medium, provided the original work is properly cited. their shoulders on the stable surface of knee height, the IO/RA ratio were higher than other positions but there were no significant difference between positions for EO/RA, IO/RA ratio.

CONCLUSION: Based on this result, using various bases of support and changing the height of bridging exercise may be used to provide effective trunk stabilization exercises.

Key Words: Bridging exercise, EMG, Trunk stabilization

\section{Introduction}

Trunk stabilization exercises provide the efficiency of movements by increasing spinal and pelvic stability in performing functional postures and exercises (GarciaVaquero et al., 2012), and are useful for maintaining balanced postures, improving muscle strength, and preventing musculoskeletal chronic diseases caused by aging (Choi et al., 2012). In addition, the simultaneous activation of trunk muscles through trunk stabilization exercises increases spinal stability by providing segmental fixation to the structure around the spine against large loads (Marshall et al., 2005; Vera-Garcia et al., 2007; Ha et al., 2013). When there is segmental instability in the spine, 
the contraction of global muscles during movements causes pressing and shearing forces, which subsequently cause major pain in the lumbopelvic region and stress on the tissues within the range of motion (ROM) (Kisner and Colby, 2012). Therefore, for trunk stabilization exercises, while the activity of each muscle during physical movements is important to provide a stable structure, the ratio of local to global muscles is duly important (Stevens et al., 2006; Hodges and Moseley, 2003).

In general, pelvic tilt exercise, abdominal draw-in maneuver, and bridging exercise, which are frequently used in clinical practice, are suggested for trunk stabilization exercises (Hubley-Kozey and Vezina, 2002). As a closed kinetic chain exercise, bridging exercise can conveniently induce the contraction of the multifidus and transversus abdominis. In addition, as this exercise improves the coordination of global muscles in the body surface and local muscles in the deep part (O'sullivan et al., 1997), it is effective not only in controlling the trunk (O'sullivan et al., 1997; Stevens et al., 2006), but also in strengthening extensors in the hip and lower limbs (Kisner and Colby, 2012). For this reason, bridging exercise is also used in rehabilitation exercises for patients with lumbar pain or rehabilitation training for sports players through various applications in clinical practice (Behm et al., 2010). In recent years, some revised methods have been suggested for the existing bridging exercise. First, postures are modified or movements are added so that the supine, lateral, and prone positions are performed during bridging exercise and the trunk muscle activity of each posture is compared (Bjerkefors et al., 2010; McGill and Karpowicz, 2009).

Another approach is to perform bridging exercise by applying unstable surfaces such as the TOGU ball, BOSU ball, and Swiss ball (Imai et al., 2010; Stevens et al., 2006; Desai and Marshall, 2010). The application of these unstable surfaces increases the activation of muscles required to maintain postural stability in the spine and trunk (Son et al., 2009; Imai et al., 2010; Czaprowski et al.,
2014). In addition, the application of resistance exercise to the limbs during bridging exercise on an unstable surface increases the activation of trunk muscles as stabilizers (Behm et al., 2005). Another method suggests the combined use of bridging exercise and the sling. As the bridging exercise applying the sling provides more unstable elements than the traditional bridging exercise, it helps perform trunk stabilization exercises by increasing the activation of local muscles located in the deep trunk (Saliba et al., 2010). In addition, the sling is effective in providing a reduction in gravity, which is a major advantage of aquatic exercise therapy. Therefore, it is used in the mobilization of joints, stretching, muscle endurance training, relaxation, and trunk stabilization through various modifications (Kirkesola, 2001).

A review of the existing studies shows that some studies researched various bridging exercise methods that include applying the internal resistance of the trunk such as combining the movements of the upper or lower limbs during bridging exercise (Marshall and Murphy, 2005; Murphy and Wilson, 1996), and combining bridging exercise with unstable surfaces such as the TOGU ball, Swiss ball, and sling by mostly applying them to the lower limbs. However, a relatively small number of studies examined the activation of trunk muscles during bridging exercise by changing the base of support for the shoulders. Therefore, the purpose of this study is to suggest effective trunk stabilization exercises by conducting a comparative analysis on the methods of changing the base of support for the shoulders in performing bridging exercise that is used as a trunk stabilization exercise.

\section{Methods}

\section{Subjects}

20 healthy volunteers in their twenties (10 men, 10 women) were recruited for the study and gave their 
informed consent to participate. Exclusion criteria for subjects were: 1) complaints of low back pain in last 6 months, 2) a history of neuromuscular or orthopaedic disorders, 3) presence of severe postural abnormality. This study was approved by the ethical commission of Catholic university of Pusan.

\section{EMG recording and data processing}

To measure the muscle activities of erector spinae (ES), rectus abdominis (RA), external oblique (EO), internal oblique (IO), EMG data was collected using a Telescan program and EMG system (LXM3204, LAXTHA Inc., KOREA). The signals were amplified with an overall gain of $1210 \mathrm{~Hz}$. The raw signal was band-pass filtered between 50 to $500 \mathrm{~Hz}$ and bandstop filters $(60 \mathrm{~Hz})$ were used. Normalization of EMG corresponding maximal EMG amplitude allows interindivisual comparison to the individual maximum.

Root mean square (RMS) was calculated for raw EMG measurement data. The MVIC test positions were performed in according to Kendall et al. (2005). The MVICs of the muscles were measured in three trials before the tasks. To obtain a measurement, EMG signal was recorded continuously for 10 s and calculated to determine the \%MVIC for middle 6s. EMG electrodes were placed at $2 \mathrm{~cm}$ laterally to spinous process of L2 for ES. For RA, $5 \mathrm{~cm}$ lateral to umbilicus; for IO, halfway between the ASIS and umbilicus; For EO, $15 \mathrm{~cm}$ lateral to umbilicus (Cholewicki and McGill, 1996).

\section{Experimental design}

The bridge exercise was performed under 5 experimental conditions (Fig. 1) : bridge exercise with their shoulders on a stable table (1/2 knee height, knee height), and on a sling (1/2 knee height, knee height), conventional bridge exercise. The height of shoulder-support surface was defined as the patellar height from floor in standing position for each subject.
Three trials for every condition were performed in a random sequence. The tasks were performed with $10 \mathrm{~s}$ durations each, 1 min resting interval. EMG measurement was performed during the middle $6 \mathrm{~s}$.

\section{Statistical analysis}

All data were analyzed using SPSS version 21.0 (SPSS, INC., USA), the level of statistical significance was set at $a=.05$. Analysis of variance (ANOVA) was used to compare the \%MVIC of the 4 muscles for 5 positions, if significance differences were detected, Tukey HSD was carried out to identify differences.

\section{Results}

\section{General characteristics}

Subject characteristics are summarized in Table 1.

Table 1. Mean $\pm S D$ of General characteristics of subjects

\begin{tabular}{lrlll}
\hline \multicolumn{2}{c}{ gender $(\mathrm{n})$} & age $(\mathrm{yrs})$ & heights $(\mathrm{cm})$ & weights $(\mathrm{kg})$ \\
\hline Men & 10 & $21.3 \pm 1.26$ & $169.7 \pm 6.20$ & $61.5 \pm 7.09$ \\
Women & 10 & & & \\
\hline
\end{tabular}

2. EMG activity of each muscle during various positions

Table 2 shows the muscle activity from EMG measurement at each position. Muscle activity in RA, EO, IO increased significantly during position 4 (shoulder on the sling of $1 / 2$ knee height).

Activation ratios of local muscle to global muscle are shown in Fig. 2. There were no significant differences in EO/RA, IO/RA ratio, but during position 3 (shoulder on the stable table), IO/RA ratio was higher than other positions. 
Table 2. Mean \pm SD of EMG activity (\%MVIC) for each position

\begin{tabular}{|c|c|c|c|c|c|c|c|}
\hline & Position 1 & Position 2 & Position 3 & Position 4 & Position 5 & $\mathrm{~F}$ & $\mathrm{p}$ \\
\hline \multirow{2}{*}{ RA } & $18.79 \pm 2.75$ & $27.10 \pm 3.03$ & $24.53 \pm 3.44$ & $45.05 \pm 6.28$ & $41.54 \pm 6.20$ & 14.60 & $.00 *$ \\
\hline & \multicolumn{7}{|c|}{ Position $1<$ Position 4,5} \\
\hline ES & $61.14 \pm 4.44$ & $55.16 \pm 3.91$ & $59.77 \pm 4.15$ & $56.27 \pm 4.51$ & $57.71 \pm 5.24$ & .69 & .60 \\
\hline \multirow{2}{*}{$\mathrm{IO}$} & $35.82 \pm 4.48$ & $41.00 \pm 4.48$ & $46.96 \pm 5.18$ & $60.75 \pm 6.17$ & $55.14 \pm 6.21$ & 6.93 & $.00 *$ \\
\hline & \multicolumn{7}{|c|}{ Position $1<$ Position 4} \\
\hline EO & $37.41 \pm 3.80$ & $45.37 \pm 4.33$ & $44.49 \pm 3.94$ & $70.13 \pm 5.39$ & $63.20 \pm 5.47$ & 17.81 & $.00 *$ \\
\hline
\end{tabular}

* RA: rectus abdominis

ES: erector spinae

IO: internal oblique

EO: external oblique

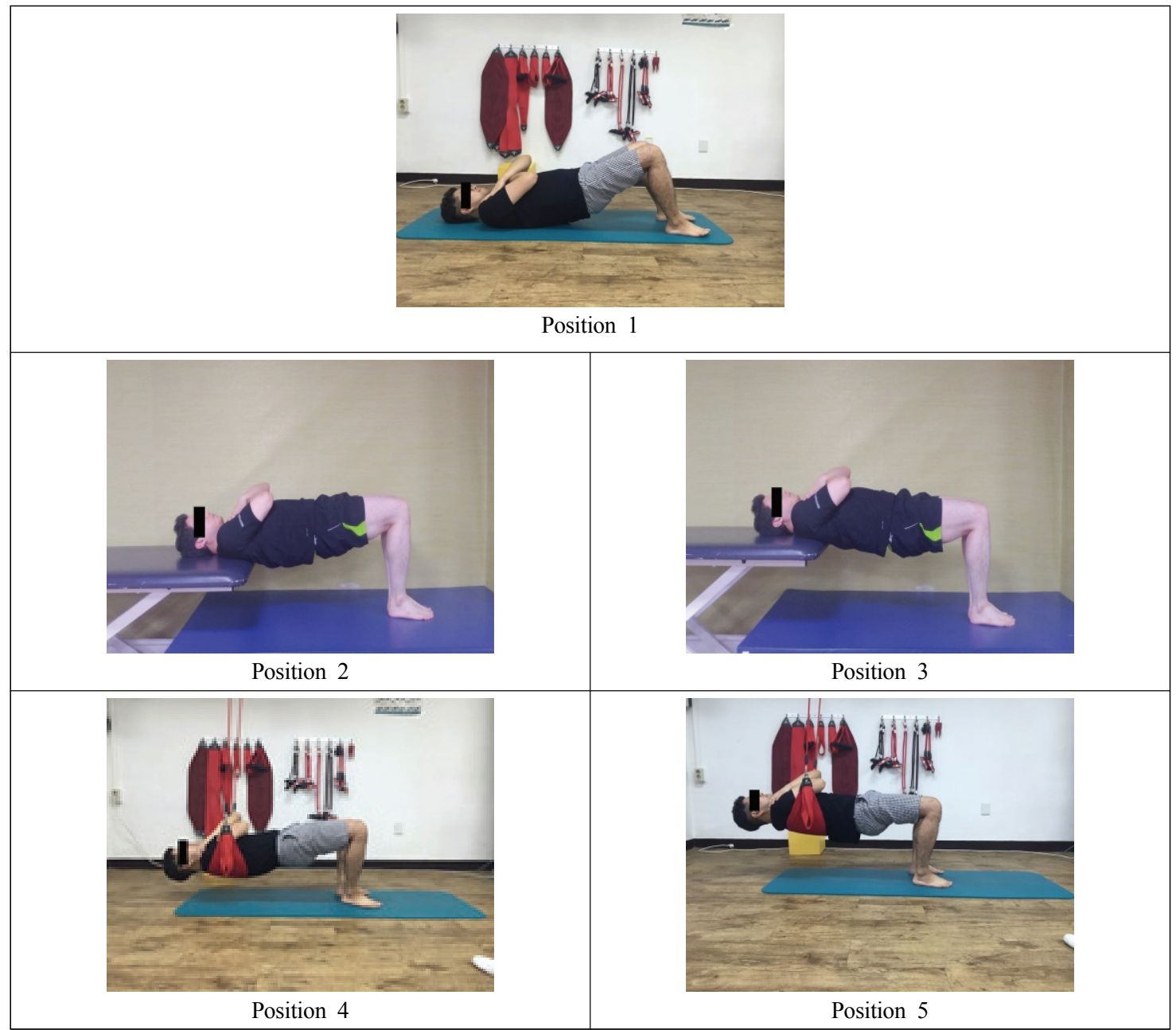

Fig. 1. experimental positions ; Position 1 : conventional bridge exercise ; Position 2 : Bridge exercise; shoulder on the stable table of $1 / 2$ knee height ; Position 3 : Bridge exercise; shoulder on the stable table ; Position 4 : Bridge exercise; shoulder on the sling of $1 / 2$ knee height ; Position 5 : Bridge exercise; shoulder on the sling 




Fig. 2. Comparison of EO / RA and IO/RA ratio between each position

\section{Discussion}

This study intended to examine changes in trunk muscle activation during bridging exercise that is commonly used for trunk stabilization when providing an unstable surface to the base of support for the shoulders or changing the height of the base of support for the shoulders. As a result, when a bridging exercise was performed or the base of support for the shoulders became unstable through the use of a sling, except the erector spinae (ES), the muscle activities of the rectus abdominis (RA), external abdominal oblique (EO), and internal abdominal oblique (IO) statistically significantly increased. When the base of support for the shoulders was adjusted to knee height on a stable surface, the IO/RA ratio increased.

As bridging exercise generates the tension of the thoracolumbar fascia and intra-abdominal pressure, it induces the contraction of the IO while maintaining segmental stability (Hodges and Richardson, 1998) and contributes to improving the stability of trunk muscles through the control of muscles around the lumbopelvic region (Akuthota and Nadler, 2004). According to the results of precedent studies, the proper activation of local muscles is required to realize optimal stability during trunk stabilization exercises (Richardson et al., 2004; Hodges et al., 1999). For the activation of local muscles, various recent studies have made attempts to change the existing bridging exercise, such as applying an unstable surface to the base of support for the legs (Stevens et al., 2006; Marshall and Murphy, 2008) and adding the movements of the limbs during bridging exercise (Kim et al., 2013).

In this study, when a sling was applied to the base of support for the shoulders during bridging exercise, except the ES, the muscle activities of the RA, IO, and EO statistically significantly increased. Marshall and Murphy (2005) conducted a study in which a Swiss ball was applied to the base of support for the legs during a trunk stabilization exercise and found a statistically significant increase in the muscle activity of the RA. They explained this as the result of a higher level of trunk muscle activity to maintain the center of the body as the Swiss ball provides instability to the body. This is similar to the result of the present study. Desai and Marshall (2010) stated that the application of unstable surfaces such as the TOGU ball and Swiss ball during stabilization exercises decreases physical balance as these surfaces increase lumbar movements compared to stable surfaces, and subsequently increases the risk of falls. However, they noted that the provision of these unstable surfaces during rehabilitation increases trunk muscle activation, and thus can be applied as a balance strategy.

In a study by Kim et al. (2013), the subjects performed additional arm movements while the Swiss ball was placed between their shoulder blades and the floor. Here, as the arm movements on the unstable surface worked as internal resistance, the activation of the IO increased. In the present 
study, when an unstable surface using a sling was applied to the base of support for the shoulders, except the ES, the muscle activities of the remaining RA, IO, and EO statistically significantly increased. Therefore, using the sling as an unstable surface may be more effective in stimulating the IO that is a local muscle. In a study by Lehman et al. (2005), they compared trunk muscle activation using a Swiss ball in various bridging exercise postures, and reported statistically significant increases in the activation of the RA and EO due to a reduction in the stability of the surface, but no statistically significant differences in the activation of the IO and ES. As a reason for this, they pointed out large variations in the activation of the IO across the study's ten subjects. This illustrated the effects of inter-experimental variations in verbal instructions and sensory feedback on muscle activation. Unlike this result, the present study did not show changes in the muscle activity of the ES, but showed statistically significant increases in the muscle activities of the RA, EO, and IO. This may be because, unlike the Swiss ball, the application of the sling activated the local muscles. A previous study reported that the application of a sling is more effective for trunk stabilization exercises than that of the existing bridging exercise or a Swiss ball because it increases the activation of local muscles of the trunk, such as the IO and transervers abdominis (Dannelly et al., 2011; Stuge et al., 2004).

Arokoski et al. (2001) stated that the activation of abdominal muscles is generally lower than the activation of muscles around the spine during bridging exercise, which is because the load generated during the bridging exercise is focused on the muscles around the spine. Therefore, to compensate for the high activation of muscles around the spine, as with the transervers abdominis and the multifidus, the IO, which is a local muscle, is involved in the segmental stability of the spine, and the activation of the RA and EO, which are global muscles, increases. As a result, this simultaneously generates posterior pelvic tilts and trunk flexion. The present study also showed that when the sling was applied, the EO/RA ratio was higher than the IO/RA ratio although there was no statistically significant difference. This coincides with the result of a study by Imai et al. (2010) that during a stabilization exercise on an unstable surface, the activation of the EO increased to control the movements of trunk flexion and rotation.

The activation ratio of local versus global muscles (e.g. IO/RA, EO/RA) is not only a sensitive discriminator of altered recruitment patterns but also a important component of a therapeutic exercise program. Marshall and Murphy (2005) noted that the analysis of the IO/RA ratio is important to learn about the performance of stabilization exercises that minimize the activation of the RA compared to other muscles in the lumbopelvic region. In the present study, when the base of support for the shoulders was set as a stable surface at knee height during bridging exercise, the highest IO/RA ratio was observed. From this aspect, as each individual posture changes muscle activity and the co-operation of muscles, during stabilization exercises, it is necessary to consider muscle ratios and use them according to the purpose of exercise, rather than to activate specific muscles.

The present study examined the effects of bridging exercise, which is commonly used in clinical practice, on trunk muscle activation when the base of support for the shoulders was changed. When the base of support for the shoulders was lifted using a sling and an unstable surface was applied, the activation of the trunk muscles RA, EO, and IO increased. In addition, when only the height of the base of support for the shoulders was changed on a stable surface, the highest IO/RA ratio was confirmed. This study has some limitations that sample size is relatively small and all the subjects were healthy adults in their twenties. And this study focused merely between bridge exercise and global muscle activation of the trunk. We did not measure the transversus abdominis and multifidus as local muscles. Therefore, various studies are required 
to examine the effects of bridging exercise on the trunk muscle activation of patients with lumbar pain when the base of support for the shoulders is changed.

\section{Conclusion}

The present study examined changes in trunk muscle activity according to changes in the stability and height of the base of support for the shoulders during bridging exercise. When the bridging exercise was performed using a sling, which was an unstable surface, at the half of knee height, the highest trunk muscle activity was shown. When a stable surface was applied at knee height, the highest IO/RA ratio was observed. Based on this result, using various bases of support and changing the height of these bases during bridging exercise is likely to help perform effective trunk stabilization exercises. Furthermore clinicians can apply adequate bridge exercise to prevent musculoskeletal disease or to improve the trunk muscle strength in clinical setting.

\section{References}

Akuthota V, Nadler SF. Core strengthening. Arch Phys Med Rehabil. 2004;85(3 Suppl 1):S86-92.

Arokoski JP, Valta T, Airaksinen O, et al. Back and abdominal muscle function during stabilization exercises. Arch Phys Med Rehabil. 2001;82(8):1089-98.

Behm DG, Drinkwater EJ, Willardson JM, et al. The use of instability to train the core musculature. ApplPhysiolNutrMetab. 2010;35(1):91-108.

Behm DG, Leonard AM, Young WB, et al. Trunk muscle electromyographic activity with unstable and unilateral exercises. J Strength Cond Res. 2005; 19(1):193-201.

Bjerkefors A, Ekblom MM, Josefsson K, et al. Deep and superficial abdominal muscle activation during trunk stabilization exercises with and without instruction to hollow. Man Ther. 2010;15(5):502-7.

Choi SH, Lim JH, Cho HW, et al. The Effects of Trunk Stabilization Exercise Using Swiss Ball and Core Stabilization Exercise on Balance and Gait in Elderly Women. J Korean Soc Phys Med. 2012;7(1):49-58,

Cholewicki J, McGill SM. Mechanical stability of the in vivo lumbar spine: implications for injury and chronic low back pain. ClinBiomech (Bristol, Avon). 1996;11(1):1-15.

Czaprowski D, Afeltowicz A, Gebicka A, et al. Abdominal muscle EMG-activity during bridge exercises on stable and unstable surfaces. Phys Ther Sport. 2014; 15(3):162-8.

Dannelly BD, Otey SC, Croy T, et al. The effectiveness of traditional and sling exercise strength training in women. J Strength Cond Res. 2011;25(2):464-71.

Desai I, Marshall PW. Acute effect of labile surfaces during core stability exercises in people with and without low back pain. J ElectromyogrKinesiol. 2010;20(6): 1155-62

Garcia-Vaquero MP, Moreside JM, Brontons-Gil E, et al. Trunk muscle activation during stabilization exercises with single and double leg support. J ElectromyogrKinesiol 2012;22(3):398-406.

Ha Y, Lee GC, Bae WS, et al. The Effect of Abdominal Muscle Drawing-In Exercise During Bridge Exercise on Abdominal Muscle Thickness, using for Real-time Ultrasound Imaging. J Korean Soc Phys Med. 2013;8(2):231-8.

Hodges P, Cresswell A, Thorstensson A. Preparatory trunk motion accompanies rapid upper limb movement. Exp Brain Res. 1999;124(1):69-79.

Hodges PW, Moseley GL. Pain and motor control of the lumbopelvic region: effect and possible mechanisms. J ElectromyogrKinesiol. 2003;13(4):361-70.

Hodges PW, Richardson CA. Delayed postural contraction of transversus abdominis in low back pain associated 
with movement of the lower limb. J Spinal Disord. 1998;11(1):46-56.

Hubley-Kozey CL, Vezina MJ. Muscle activation during exercises to improve trunk stability in men with low back pain. Arch Phys Med Rehabil. 2002;83(8): 1100-8.

Imai A, Kaneoka K, Okubo Y, et al. Trunk muscle activity during lumbar stabilization exercises on both a stable and unstable surface. J Orthop Sports Phys Ther. 2010;40(6):369-75.

Kendall FP, McCreary EK, Provance PG, et al. Muscle: Testing and Function, with Posture and Pain $\left(5^{\mathrm{TH}}\right.$ ed). Balttimore, MD. Lippincott Williams \& Wilkins. 2005.

Kim MJ, Oh DW, Park HJ. Integrating arm movement into bridge exercise: Effect on EMG activity of selected trunk muscles. J ElectromyogrKinesiol. 2013;23(5): $1119-23$

Kirkesola G. Sling Exercise Therapy (S-E-T) : A total concept for exercise and active treatment of musculoskeletal disorders. Korean J Orthop Manu Ther. 2001;7(1): 87-106.

Kisner C, Colby LA. Therapeutic exercise : foundations and techniques $\left(6^{\mathrm{TH}}\right.$ ed). Philadelphia, PA: F.A. Davis. 2012.

Lehman GJ, Hoda W, Oliver S. Trunk muscle activity during bridging exercises on and off a Swiss ball. ChiroprOsteopat. 2005;13:14.

Marshall PW, Murphy BA. Core stability exercises on and off a Swiss ball. Arch Phys Med Rehabil. 2005;86(2):242-9.

Marshall PW, Murphy BA. Muscle activation changes after exercise rehabilitation for chronic low back pain. Arch Phys Med Rehabil. 2008;89(7):1305-13.

McGill SM, Karpowicz A. Exercises for spine stabilization: motion/motor patterns, stability progressions, and clinical technique. Arch Phys Med Rehabil. 2009;90(1):118-26.

Murphy AJ, Wilson GJ. Poor correlations between isometric tests and dynamic performance: relationship to muscle activation. Eur J ApplPhysiolOccup Physiol 1996;73(3-4):353-7.

O'Sullivan PB, Phyty GD, Twomey LT, et al. Evaluation of specific stabilizing exercise in the treatment of chronic low back pain with radiologic diagnosis of spondylolysis or spondylolisthesis. Spine. 1997; 22(24):2959-67.

Richardson C, Hodges PW, Hides J, et al. Therapeutic exercise for lumbopelvic stabilization: a motor control approach for the treatment and prevention of low back pain $\left(2^{\text {nd }}\right.$ ed). Edinburgh U.k. Churchill Livingstone. 2004.

Saliba SA, Croy T, Guthrie R, et al. Differences in transverse abdominis activation with stable and unstable bridging exercises in individuals with low back pain. $\mathrm{N}$ Am J Sports Phys Ther. 2010;5(2):63-73.

Son ST, Kim MH, Kim HJ, et al. The Comparison of Trunk Muscles Activity During Bridging Stabilization Exercises on Swiss Ball According to Change of Position. J Korean Soc Phys Med. 2009;4(4):221-9.

Stevens VK, Bouche KG, Mahieu NN, et al. Trunk muscle activity in healthy subjects during bridging stabilization exercises. BMC MusculoskeletDisord. 2006;7:75.

Stuge B, Laerum E, Kirkesola G, et al. The efficacy of a treatment program focusing on specific stabilizing exercises for pelvic girdle pain after pregnancy: a randomized controlled trial. Spine. 2004;29(4):351-9.

Vera-Garcia FJ, Elvira J, Brown SH, et al. Effects of abdominal stabilization maneuvers on the control of spine motion and stability against sudden trunk perturbations. J ElectromyogrKinesiol. 2007;17(5):556-67. 